GRADIATION\&APPLICATIONS

ISSN 2466-4294 (online) | rad-journal.org

Vol. 3 | Issue 2 | pp. 98-105, 2018

doi: 10.21175/RadJ.2018.02.016

Original research paper

\title{
THE SPES FACILITY AS AN INTENSE NEUTRON SOURCE: RADIATION RESISTANCE OF POLYMERIC MATERIALS AND RESIDUAL ACTIVATION CALCULATIONS*
}

\author{
Matteo Ferrari ${ }^{* *}$, Antonietta Donzella ${ }^{1}$, Aldo Zenoni ${ }^{1}$, Andrea Avanzini' \\ Davide Battini ${ }^{1}$, Fabio Bignotti ${ }^{1}$, Giorgio Donzella ${ }^{1}$, Stefano Pandini ${ }^{1}$, \\ Alberto Andrighetto ${ }^{2}$, Lisa Centofante ${ }^{2}$, Stefano Corradetti ${ }^{2}$, Michele Ballan ${ }^{2}$, \\ Francesca Borgna², Fabio D'Agostini², Mattia Manzolaro², Alberto Monetti², \\ Massimo Rossignoli ${ }^{2}$, Daniele Scarpa ${ }^{2}$, Davide Turcato ${ }^{2}$
}

${ }^{1}$ Department of Mechanical and Industrial Engineering, University of Brescia, Brescia, Italy ${ }^{2}$ National Laboratories of Legnaro of INFN, Legnaro (Pd), Italy

\begin{abstract}
SPES is a new generation ISOL facility for the production of intense Radioactive Ion Beams by fission reactions at high rate. Two main topics related to the management of SPES as an intense neutron source are here discussed: the radiation resistance of polymeric components used for its construction and the residual activation of the system after machine shutdown. Radiation effects on elastomeric O-rings and lubricating grease are experimentally investigated to assure reliable operation of the facility and safe post-operation management. Experimental protocols have been developed to irradiate samples in a neutron and gamma facility of a TRIGA Mark II nuclear research reactor. Based on the results of post-irradiation mechanical tests, the most radiationresistant products are selected. A case study is dedicated to the life prediction of the O-ring of a SPES gate valve. Moreover, extensive Monte Carlo calculations are performed to evaluate the residual radioactivity of the facility after operation. The outcomes represent useful inputs to plan inspection and maintenance during the facility shutdown.
\end{abstract}

Key words: Component failure, ISOL facility, Monte Carlo calculations, neutron activation, neutron damage, $O$-ring prediction map, radiation resistance, polymeric materials, SPES

\section{INTRODUCTION}

The selective production of exotic species (SPES) project is a new facility to produce radioactive ion beams (RIBs) using the isotope separation on line (ISOL) technique [1]. SPES is currently under construction at Legnaro National Laboratories (LNL) of the Italian Institute of Nuclear Physics (INFN). A multi-foil uranium carbide target is impinged by a $40 \mathrm{MeV}, 200 \mu \mathrm{A}$ proton beam generated by a cyclotron proton driver. In these conditions, a fission rate of approximately $10^{13}$ fissions per second is expected in the target. The ${ }^{238} \mathrm{U}$ fission fragments produced in this way are delivered to the ion source making use of a tubular transfer line. Here they can be ionized (at charge state $1+$ ) and subsequently accelerated toward the experimental areas [2] [3]. In ISOL facilities the target system can be combined with different types of ion sources to optimize the production of specific radioactive ion beams. The SPES target system is composed of seven coaxial uranium carbide disks closed inside a graphite box. It is closed under the vacuum inside a water-cooled chamber and has to maintain high working temperature close to $2000^{\circ} \mathrm{C}$. During the operation, the proton beam provides the heating power required to keep the target at the desired temperature. To avoid undesired system overheating, its parameters must be strictly controlled. Extensive research is currently carried out to develop such a challenging and innovative system.

\subsection{The management of SPES as an intense neutron source}

The development of a second generation ISOL facility like SPES requires the solution of several previously unexplored nuclear management issues. Intense neutron and photon fields expected in operation phase can damage the materials employed for the construction of the Target System and of its neighborhoods. In particular, non-metallic components are the most sensitive to ionizing radiation [4]. Despite their sensitivity, polymeric components as elastomeric O-rings and lubricating greases are necessarily employed. Severe radiation-induced modifications of their mechanical and structural

\footnotetext{
* This paper was presented at the Sixth International Conference on Radiation and Applications in Various Fields of Research (RAD 2018), Ohrid, Macedonia, 2018.

matteo.ferrari@unibs.it
} 
properties are expected, possibly compromising the whole facility operation.

After the operation, the whole system maintains very high levels of residual radioactivity. Activation is induced both by primary protons and by neutrons produced in operation. Exhausted target systems at the end of their life cycle must be removed from the rest of the system. Due to their high radioactivity, they must be transferred to a shielded temporary storage by using a completely automatic handling system. Complete isolation from the outer environment has to be maintained for the whole storage time, to prevent the release of radioactive contaminants in the environment. Isolation relies on the sealing ability of the polymeric elements, whose performance can be compromised by radiation damage and by viscoelastic material behavior.

The residual activation of the rest of the structures requires particular care in planning inspection and maintenance operations during machine shutdown in safe conditions.

All the mentioned radiation-induced effects must be carefully evaluated to grant safe and steady SPES operations. For this reason, dedicated experimental and computational studies are carried out in the framework of the cooperation between SPES and the University of Brescia, Italy.

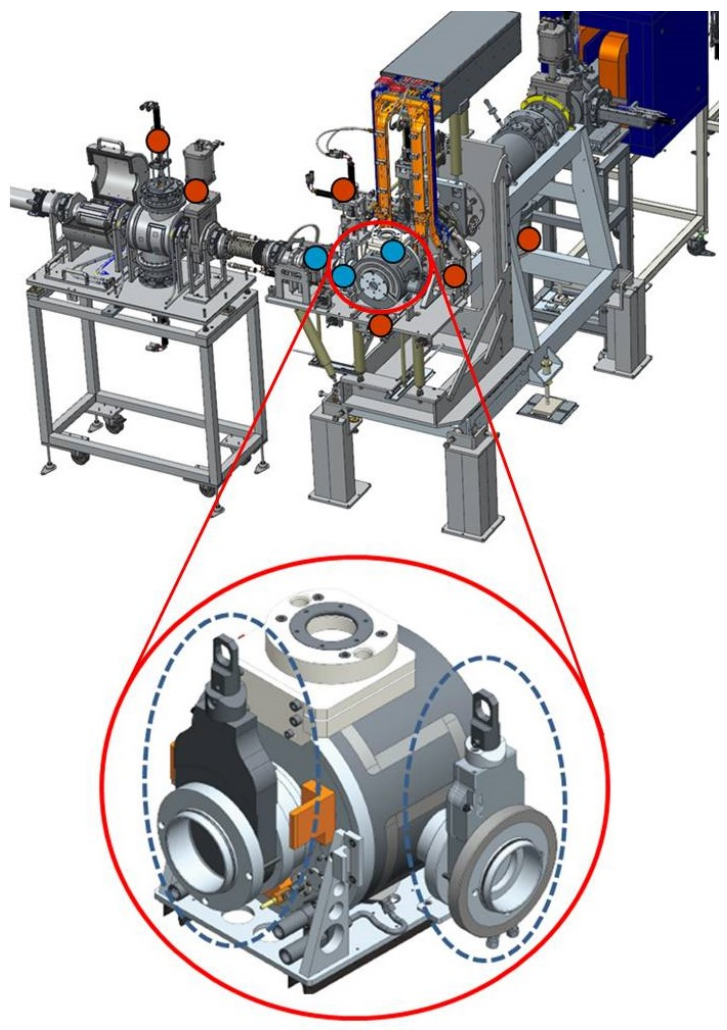

Figure 1. A 3D model of the SPES facility. Top: part of the proton line (Left) and of the RIB channel (Right) are present. The positions where elastomeric O-rings and lubricants are used are evidenced by blue and red dots respectively. Bottom: the TIS unit is represented. The gate valves equipped with Orings are evidenced by dotted lines.

\subsection{The SPES Target System and its life cycle}

The core of the SPES system is represented by the Target and Ion Source (TIS) complex (see Figure 1). It houses the target and the ion source in a vacuum environment. The polymeric components required for its operation are here described. These components have a crucial role for post-service handling and storage procedures as well. The most critical components of the complex are vacuum O-rings. They have the important task of keeping the necessary high vacuum environment during operation. For precautionary purposes, the TIS uptime is limited to 15 days [3]. During this time, the O-rings are exposed to a mixed neutron and gamma fields. Neutrons originate from fission reactions and from spallation reactions induced by the primary proton beam. The fast neutron component is dominant, because neutrons are not subjected to significant moderation processes in the target and its surroundings [8]. As a reference, the most exposed EPDM-based O-ring of the TIS absorbs during a 15-day long operation a total dose of about 0.7 MGy. The neutron component of this dose is about o.6 MGy, being $85 \%$ of the total. The remaining $15 \%$ is represented by gamma dose, mainly due to fission prompt gammas. More details about dosimetry can be found in [5]. The TIS life cycle includes a 15-day long post-service cooling time before removal from the facility to reduce the residual activity.

The TIS is connected to the proton line and to the RIB channel by use of two gate valves, which are equipped with O-rings (see Figure 1). At the end of the TIS lifetime, the valves are used to isolate the TIS from the rest of the Front-End. Exhausted TIS units are placed in a temporary storage for the following five years. After this period, the residual activity level will be low enough to allow final disposal and recycling. The gate valve O-rings are crucial in preventing the release of radioactive contaminants during storage time as well During the 15-day long cooling phase and during the following 5-year long storage, the O-ring absorbs an additional gamma dose lower that $5 \mathrm{kGy}$ due to the residual radioactivity of the TIS [8]. The automatic handling of the exhausted TIS relies on the correct operation of several moving mechanical devices as pneumatic motors, screws and several kinds of bearings [6]. These components require proper lubrication. For example, the bearings necessary to mount and dismount the Target System, which are placed below the TIS (see Figure 1) are lubricated with grease. These components are not removed with the TIS and their lifetime is about 7-year long. Integrated neutron doses absorbed during this time range between 25 MGy and 30 MGy, being much higher than the ones expected for the O-rings.

Radiation-induced modifications of O-rings and lubricants can lead to TIS failure during the operation, to the failure of the automatic handling system and to the release of radioactive contaminants during storage. For these reasons, experimental protocols for the radiation-damage evaluation have been developed. Based on post-irradiation examinations, procedures to define safe usability conditions for components to be specifically used in the SPES project have been developed. 
2. IRRADIATION AND TESTING PROTOCOLS FOR POLYMERIC COMPONENTS

\subsection{Irradiation facility and dosimetry calculations}

Neutron damage studies on polymeric materials are presently very scarce in the literature. We developed protocols for irradiation and testing of elastomeric Orings [5] and lubricating greases [7]. They aim at the selection of radiation-resistant products to be used for the SPES construction.

Samples are irradiated in an in-core facility of the TRIGA Mark II nuclear research reactor of the University of Pavia, Italy (see Figure 2). All the irradiations where performed with the reactor operating at the nominal power of $250 \mathrm{~kW}$. In this condition, the total neutron flux in the facility is $1.7210^{13} \mathrm{n} / \mathrm{cm}^{2} \mathrm{~s}$. The fast neutron flux is $3.810^{12} \mathrm{n} / \mathrm{cm}^{2} \mathrm{~s}$. It includes energies higher than 0.5 $\mathrm{MeV}$. Samples are irradiated in air atmosphere at atmospheric pressure. The temperature in the facility during operation ranges between $50^{\circ} \mathrm{C}$ and $70^{\circ} \mathrm{C}$.

In the present work, the neutron and gamma dose delivered to the irradiated samples are calculated with a $\mathrm{MCNP}_{5}$ [10] Monte Carlo model of the reactor. Version 2.7.0 of the code is used. Cross-section library endf6o is used. The model was validated by an extensive campaign of neutron flux measurements previously performed [14]. No additional measurements were completed during the present work.

The neutrons in the Central Thimble facility originate from fission reactions occurring in the reactor core. Two main components of the neutron energy spectrum are present: a fast component and a thermal component which originates from the moderation of fast neutrons due to the materials present in the reactor core. More details can be found in [5]. Fast neutrons are particularly effective in delivering dose to polymeric materials. In fact, the most important process of energy transfer is elastic scattering on light nuclei, in particular on hydrogen. For this reason, we consider the fast component of the neutron spectrum present in the Central Thimble as a good approximation of the fast neutron component generated by SPES. Similar processes of neutron energy transfer to polymers are expected in both the Central Thimble facility and in the SPES TIS. The possibility of using the Central Thimble to replicate the radiation fields expected in operation is extensively discussed in [8].

The neutron dose is highly dependent on the hydrogen content. For this reason, carbon, hydrogen and nitrogen composition is measured for all the irradiated samples with $\mathrm{CHN}$ analysis. Results are used to model the irradiated materials in the simulations. The majority of the tested products have a hydrogen concentration ranging between $7 \%$ and $14 \%$ of the total mass [5]. The neutron component of the dose ranges accordingly from $62 \%$ to $70 \%$. The FPM-based O-ring material is saturated with fluorine and contains only about $1 \%$ of hydrogen [5]. The neutron dose component for this material is about a factor four lower compared to the one of the other EPDM-based materials. Dosimetry in a mixed neutron and gamma field is a complex topic that cannot be extensively developed in this paper. A more complete approach can be found in [5] and [8]. Neutron and photon dose rates are calculated for O-rings and greases in their specific SPES application as well.

The O-rings and grease are irradiated up to total doses ranging between 0.1 MGy and 9 MGy. Doses are delivered to the samples in accelerated way. The dose rate in the Central Thimble is about 500 times higher than the one expected in SPES operation. Integrated neutron doses absorbed by the samples are comparable to the ones expected in the operation. The evolution of the most relevant mechanical and structural quantities of the irradiated samples is measured as a function of the dose. The developed protocols allow the radiation stability of the analyzed materials to be comparatively evaluated, leading to the best choice for SPES applications.

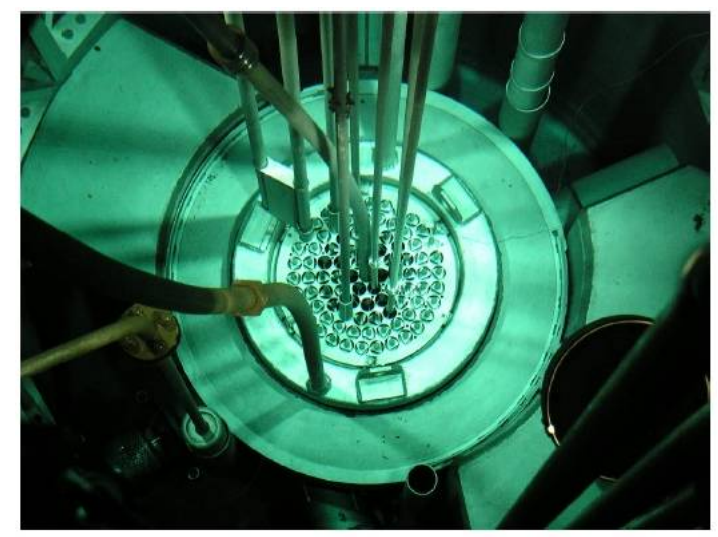

Figure 2. The core of the TRIGA Mark II nuclear research reactor of the University of Pavia, Italy. The facility used for

irradiations is the pipe reaching the middle of the core.

\subsection{Radiation damage on elastomeric $O$-rings}

Four commercially available elastomeric compounds, one FPM-based and three EPDM-based, are selected analyzing the market and the previous suppliers of the LNL. One EPDM-based product has been tested to radiation and is declared as radiation resistant by the producer. The main features of the selected products are summarized as follows:

- FPM: generic fluoroelastomer purchased by 5 Pascal Co. (Italy), one of the suppliers of the LNL laboratories. For vacuum application.

- EPDM-based product from UTS Co. (Italy), sulphur cured. For generic applications.

- EPDM Perox 70 by Dichtomatik Co. (Germany), peroxide cured. It features excellent mechanical properties as certified by third party laboratories. Standard price.

- EPDM-based Shieldseal $\AA 663$ by James Walker Co. (UK), carbon-black reinforced, peroxide cured, formulated for application in radiation environments. Certified as special material. Tested up to $1.6 \mathrm{MGy}$ of gamma radiation. Rather expensive.

More information is reported in [5] and [15]. 
Samples of these products are irradiated up to about 4 MGy of absorbed dose and submitted to standard post-irradiation examinations. Standard mechanical properties as elongation at break, elastic modulus and tensile strength are measured via tensile test. Compression set test is performed as well. Radiation-induced modifications at the structural level are investigated with Differential Scanning Calorimetry (DSC), Dynamic Mechanical Thermal Analysis (DMTA) and swelling tests. The irradiation and testing protocol and the collected results on mechanical quantities are reported in [5].

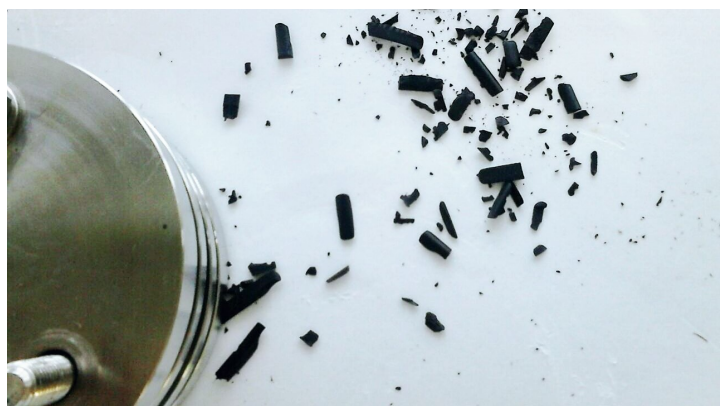

Figure 3. Complete structural failure of EPDM O-ring samples after post-irradiation compression set test. Samples irradiated at about 4 MGy of absorbed dose experience huge radiationinduced brittleness.

Results collected on all the tested materials evidence a remarkable embrittlement at the mechanical level (see Figure 3) and a significant radiation-induced cross-linking at the structural level. In particular, elongation at break degrade significantly as a function of the dose for all the selected products. The FPM-based material shows significant embrittlement at about 1 MGy of absorbed dose. The three EPDM-based materials show different behaviors: EPDM by UTS Co. feature large variations of compression set as a function of the dose. EPDM Perox

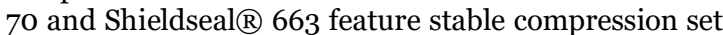
up to about 2.2 MGy.

Solvent swelling tests performed on FPM-based product and on EPDM-based product by Dichtomatik, evidence an important and progressive decrease of the swelling ratio as a function of the absorbed dose [15]. This is an indicator of an increased cross-linking of the polymer at the structural level.

Despite the significance in the radiation hardness viewpoint, precise indications about the usability conditions of the irradiated materials are not provided by these outcomes. Based on the collected results, EPDM-based Perox 70 by Dichtomatik is chosen for further investigation.

\subsection{Life prediction of SPES gate valve O-rings}

The working life of a component exposed to intense radiation fields has to be determined to define reliable operation conditions. The working life is defined for a specific component using damage thresholds referred to as endpoints. Endpoints are related to the modification of mechanical and structural parameters that are significant for the specific operation of the component. They depend on the functional limits of the component in the specific system. A methodology for the estimation of the working life of the Dichtomatik EPDM-based O-rings equipping the SPES gate valves (see Figure 1) is here proposed.

Two main effects are considered for the design of gate valves working in intense neutron fields. Structural failure is related to a radiation-induced brittle failure of the O-ring due to increased fragility, as shown in Figure 3. Functional failure is related to the loss of sealing due to a radiation-induced reduced contact pressure between the O-ring and the groove where it is installed. Several experimental and computational steps are required to define both structural and functional endpoints.

A complete characterization of the material as a function of the absorbed dose is required. Experimental Vacuum Leak Tests (VLT) are performed to measure the sealing ability of irradiated O-rings. Tests are realized using a Helium Leak Detector. Six different groove depths are investigated to measure the O-ring sealing performance, ranging between almost no-compression conditions to about $20 \%$ squeeze degree. Tested O-rings are irradiated at three different dose levels, ranging from 0.7 MGy to $3.55 \mathrm{MGy}$. A leakage threshold of $510^{-7} \mathrm{mbar} \mathrm{l} / \mathrm{s}$ is experimentally established according to the test outcomes. This threshold allows the minimum necessary squeeze degree to be identified. More details about the performed tests are reported in [8]. Finite element models (FEM) are used to simulate the VLT test and the gate valve in operation. The FEM model relies on the experimental characterization of the irradiated and non-irradiated EPDM samples. As a final output, the expected behavior of the gate valve O-rings is foreseen. A life prediction map is produced, as a function of three parameters: the total amount of absorbed dose, which is proportional to the service time, the chosen mechanical conditions for the O-ring and the storage time after service. As useful output, a safe usability area for operation and post-operation storage is defined. It is worth noting that the O-ring viscoelastic behavior can lead to functional failure even months or years after irradiation. The life prediction maps originate from a combination of both experimental and computational studies.

A sample of life prediction map is reported in Figure 4. The safe area and the failure areas associated to the analyzed endpoints are represented as a function of the service time and of the squeeze degree. The functional failure endpoint is associated to a 5-year long post-service storage. An increase of the service time leads to a higher risk of structural fracture. It is worth noting that the prediction capability of the map is based on several assumptions. As an important assumption, the testing irradiation conditions in the reactor facility are considered comparable to the operation ones in damaging the O-rings, given the same total dose. However, the radiation dose in the reactor facility is delivered in accelerated way compared to expected operation conditions. Therefore, the position of the failure endpoint in the map could vary accordingly. For this reason, the maps provide general indications of the expected trends and have to be interpreted consequently. 


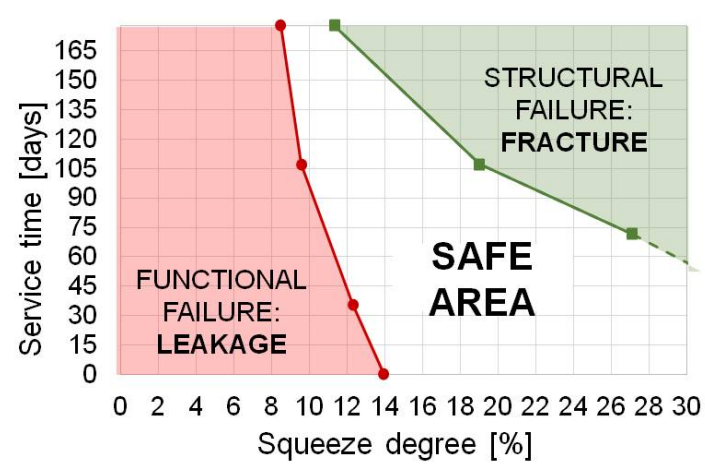

Figure 4. A Life prediction map for the SPES gate valve EPDM-based O-ring by Dichtomatik is shown. Functional failure, structural failure and safe area are represented in red, green and white respectively. The represented areas depend on the service time (proportional to the absorbed dose) and on the O-ring compression in its seat for a fixed 5-year long post-operation storage. The map results from a combination of both experimental and computational studies.

As a general thumb rule for the prevision map interpretation, the larger the safe area, the better. The case analyzed in Figure 4 supports the precautionary choice of a 15-day long TIS uptime. The map provides useful service and storage limits for the SPES TIS to avoid both functional and structural failure. A squeeze degree range is suggested for safe application. A complete description of the developed methodology and of the assessed results is reported in [8].

\subsection{Radiation damage on lubricating greases}

Seven commercially available lubricating greases are selected from some of the most relevant producers worldwide. Two products have been tested to radiation and are declared as radiation resistant by the producer. The main features of the selected products as declared by the producer are listed as follows:

- AFB-LF grease by THK Co. (Japan) is realized with a mineral base oil and a Li based thickener. It features extreme pressure resistance and high mechanical stability.

- FAG Arcanol LOAD 220 by Schaeffler (Germany) is realized with a mineral base oil and a mixed thickener. It features high load resistance and durable performance.

- Grizzlygrease No.1 by Lubricant Consultant $\mathrm{GmbH}$ Lubcon (Germany) is realized with a mineral base oil and a $\mathrm{Li} / \mathrm{Ca}$ special soap. It is declared as radiation resistant up to $1.2 \mathrm{MGy}$ of gamma dose by the producer.

- Klüberlub BE 41-542 by Klüber Lubrication (Germany) is realized using a mineral base oil and a special Li soap. It is developed for highload rolling bearings and features extreme pressure resistance.

- Petamo GHY $133 \mathrm{~N}$ by Klüber Lubrication is realized using a mineral oil and a polyurea thickener. It is a long-term and hightemperature product.
- RG-42R-1 by Moresco Corporation (Japan) is realized using a polyphelylether base oil and a polycarbonate thickener. It is declared as radiation resistant to gamma radiation up to a very high dose value of $15 \mathrm{MGy}$.

- Turmopolgrease 2 by Lubricant Consultant $\mathrm{GmbH}$ Lubcon is realized with a polyglycol base oil and a lithium-based soap. It features a very long lubrication service.

Grease samples are irradiated up to about 9 MGy of absorbed dose in the Central Thimble facility and submitted to standard post-irradiation examination. Consistency is the most used and relevant quantity to characterize the mechanical behavior of greases [9]. It is expected to exhibit a complex behavior as a function of the dose because of its multi-phase structure [4]. Consistency is measured using a penetrometer whose tip penetrates the flat surface of the irradiated grease sample (see Figure 5). According to the consistency modifications as a function of the absorbed dose, greases can be divided in two main categories.

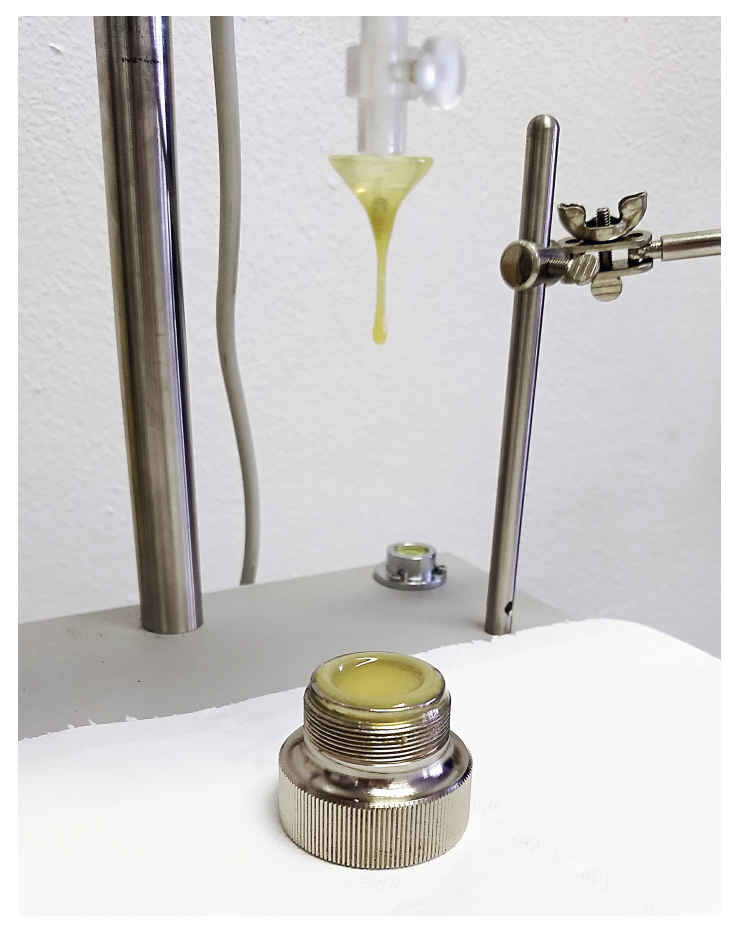

Figure 5. Post-irradiation consistency test on one of the tested greases. Radiation-induced fluidization can be observed. The fluid grease drips from the penetrometer. The picture represents an out-of-scale consistency value.

Two out of the seven tested greases (Petamo GHY $133 \mathrm{~N}$ and RG-42-R) show stable consistency values up to about $9 \mathrm{MGy}$. Five of the tested greases show extreme consistency modifications up to values exceeding the maximum instrument range. The irradiated grease in these conditions is a completely different material if compared with the non-irradiated one. Radiation-induced modifications associated to a so large consistency variation are very severe. Greases become almost fluid and drip from the testing instruments during consistency tests (see Figure 5). 
M. Ferrari et al., The SPES facility as an intense neutron source..., Rad. Applic., 2018, 3, 2, 98-105

Fluidization effects occur at dose values ranging from about 0.5 MGy to about 5 MGy. Such a dramatic grease modification can affect the operation of the lubricated bearings used in SPES. The correct removal of the exhausted TIS and its automatic handling to the temporary storage can be compromised. Expected doses to be absorbed by greases in operation are very high, reaching up to $30 \mathrm{MGy}$. For this reason, the radiation damage on lubricants is particularly challenging for the SPES design. More information about the irradiation and testing protocol and about the collected results is reported in [7].

\section{RESIDUAL ACTIVITY CALCULATIONS}

Monte Carlo simulations are a useful tool to predict the radiation fields produced by SPES during operation and their transport. The primary protons and the neutrons produced in operation generate activation while interacting with the TIS unit and with the rest of the system. The correct estimation of the system residual activity after shutdown is fundamental to plan safe management of the exhausted TIS and of the rest of the facility in the radiation safety viewpoint. To this purpose, two detailed models of SPES have been realized with the Monte Carlo codes MCNPX [10] [11] and FLUKA [12]. MCNPX can be combined with the nuclide evolution code CINDER [13] to simulate neutron and proton induced activation. Particular attention is dedicated to the simulation of the primary beam.

\subsection{Residual activity of the TIS Unit}

Exhausted TIS units are extracted from the SPES Front-End and temporarily placed in a shielded storage. A new exhausted TIS is added to the storage every 30 days. Exhausted TIS units are foreseen to be stored for 5 years. The storage contains a maximum of 60 units.

The residual activation of an exhausted TIS at the end of its life cycle has been previously calculated. Due to the very high activity level, reaching about $10^{13} \mathrm{~Bq}$, the use of a completely automatic handling is required [3] [6]. The decay of the exhausted TIS activity as a function of the cooling time is estimated in the present study. Activity levels are used to design the shielding for the exhausted TIS temporary storage. A multi-step calculation accounts for all the TIS present in the storage, each one having a different level of residual activity (see Figure 6). The thickness and the composition of the storage shielding are chosen based on these results.

During the first SPES production phases, the facility will work at lower intensities using different targets. A semi-automatic handling system for exhausted TIS produced during these phases is designed. It requires the presence of a vehicle driven by a human operator. Due to the residual TIS activity, the operator position needs to be properly shielded. Simulation outputs are useful to design the required shielding and to define the procedures to be followed.

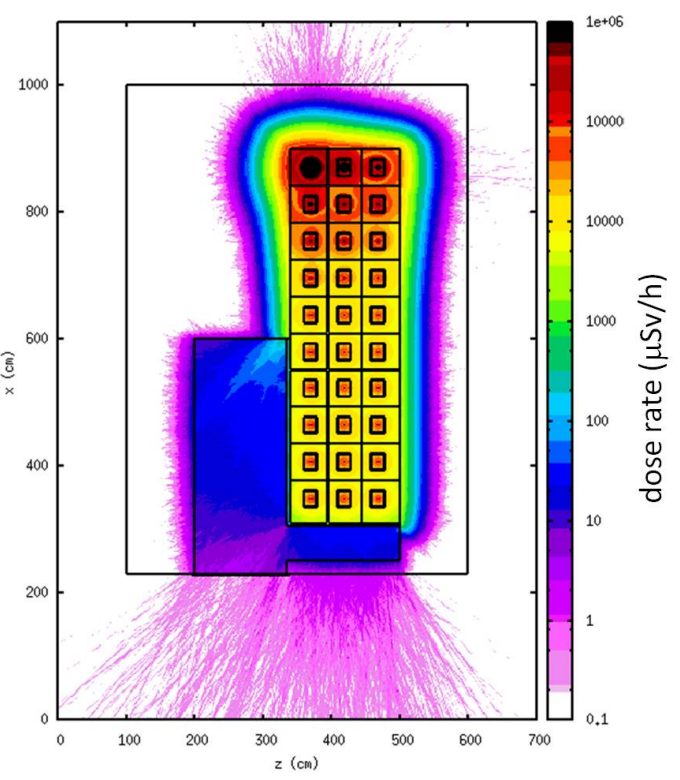

Figure 6. A multi-source Fluka simulation of the exhausted TIS in the temporary storage. A top view of the dose rate generated in the environment by 60 TIS units each one having a different level of residual activity is represented. The 30 most active TIS units can be seen, being on the same floor The most active TIS unit is placed in the top left corner. The attenuation of the dose rate is due to the introduced shielding.

\subsection{Residual activity after TIS removal}

After TIS removal, the SPES Front-End maintains relevant levels of proton and neutron activation. Bunker inspection activities are planned after every TIS removal. During these inspections, a human operator will enter the bunker to get an overview of the facility conditions. In addition, maintenance of specific elements of the proton line and of the RIB channel could be required. An accurate estimate of the residual activity of the system is necessary to plan inspection and maintenance activities in a safe way.

The SPES Front-End has a lifetime of about 7 years. For this reason, the residual activation of its elements increases as a function of the number of the completed TIS cycles, which are 15-day long. Calculations for different operation scenarios are needed. Single and multiple operation cycles followed by cooling times of different durations need to be simulated.

A first study of the residual SPES activity with MCNPX and CINDER is reported in [11]. The study evidences the presence of critical elements whose activity levels are dominant. For example, the collimators experience relevant activation induced by primary protons that are stopped in their structure. For this reason, a proper shielding for the collimators system has been designed. The results of this study are relevant for a more efficient and safe Front-End design in the viewpoint of the planned maintenance operations. In particular, the design of the proton line is being updated according to the evidenced critical items. 
A further extensive Monte Carlo study is being made to improve the results previously collected. CINDER code for nuclide evolution evaluation and FLUKA adopt very different methodologies to calculate material activation in the SPES models. Since the energy of the impinging beam and the target typology make SPES an innovative system, a benchmark between the two models focused on the residual activation is being made.

\section{Discussion}

Remarkable radiation-induced modifications of the mechanical and structural properties of elastomeric components and lubricating greases are observed after neutron and gamma irradiation. These modifications could compromise the function of O-rings and lubricants both during operation and during after service TIS handling and storage. Standard mechanical tests are completed on irradiated product samples. The evolution of all the tested mechanical parameters as a function of the dose was taken into account to compare the selected products. Based on a comparative evaluation, EPDM Perox 70 is recommended for application in SPES. Despite the lack of any radiation resistance declaration, it features satisfactory stability of its main mechanical properties as a function of the dose. The collected results give evidence that different EPDM-based O-rings can feature very different mechanical properties, as well as a very different evolution as a function of the dose. This supports the need of selecting and testing specific commercially available products. Moreover, the choice of reliable companies that can grant a sufficiently stable composition of their product over time is fundamental as well [5][8].

In a very simplified picture, two competitive phenomena are expected when radiation interacts with polymers at the structural level: cleavage and crosslinking of the polymer chains. Structural investigations were performed on two chosen products: the FPMbased O-rings and the EPDM-based ones by Dichtomatik. They both evidence an increase in the cross-linking with dose as the prevailing observed phenomenon. This can be correlated with a stiffer behavior of the products which is observed at the mechanical level. It is interesting to note that both the selected elastomeric materials experience similar modifications at the structural level, despite the different composition. An increased cross-linking could be the reason for the embrittlement reported at the mechanical level.

EPDM Perox 70 by Dichtomatik is chosen for a case study dedicated to the TIS gate valves. The experimental and computational results of this study allow a life prediction map for the specific component to be produced. The map evaluates the conditions of structural and functional failure for the specific EPDMbased O-ring in the specific installation as a function of three different parameters: the total dose absorbed in operation, the storage time and the mechanical compression of the O-ring. Safe usability conditions confirm the need to limit the TIS lifetime to 15 days for precautionary purposes. Moreover, the squeeze degree needed to maintain sealing conditions during storage is determined. This is necessary to ensure correct sealing and to avoid the release of contaminants in the environment during post-service TIS storage [8].

Concerning greases, five of the selected products experienced a complete radiation-induced fluidization. One of the greases declared as radiation-resistant to gamma radiation up to $1.2 \mathrm{MGy}$ of dose experience a complete fluidization at the same dose level delivered in the Central Thimble facility. The fluidization of the grease is probably due to the radiation-induced cleavage of the thickener structure. Two greases, Petamo GHY $133 \mathrm{~N}$ and RG-42R-1, show stable consistency values as a function of the dose. It is interesting to note that while one of the products is developed to be radiation-resistant, the other one has no declaration of radiation resistance. Moreover, the two products have very different chemical composition.

Complete models of the SPES facility have been realized using the Monte Carlo codes MCNPX and FLUKA. By the use of these models, the residual activity of the exhausted TIS unit and of the rest of the Front-End after operation is calculated. The shielding of the temporary storage of the exhausted TIS units is designed using these results. In the storage, a maximum of 60 TIS units will be stored at the same time, each of them having different levels of residual activity. This situation is simulated using a complex multi-step and multi-source model.

Exhausted TIS activity results are successfully used for the design of a semi-automatic vehicle for TIS removal during first operation phases. Since the vehicle is driven by a human operator, a proper shielding is needed. Shielding dimensions and composition are designed taking into account the residual TIS activity.

Residual activation calculations after TIS removal are completed using MCNPX and CINDER. Results allow the necessary inspections and maintenance operations during machine shutdown to be properly designed. Moreover, the design of some significant elements of the Proton Line as the collimators is updated according to the performed analyses [11].

\subsection{Further developments}

Radiation damage on polymeric materials depends on several irradiation conditions as the diffusion rate of oxygen into the rubber structure of EPDM and the dose rate. For this reason, dose rate investigations are being made on the selected EPDM. Results will be useful for a better prediction of the damage of the irradiated components during SPES operation.

Concerning greases, other radiation-induced effects as gas development and acid products developments are being investigated. A case study for the design of radiation-resistant bearings for SPES is being made.

Concerning the computational activities, a benchmark between different codes describing the SPES residual activity is being made. The comparison will provide interesting information about the description of the SPES system by different computation tools. After successful benchmark, the models will be used to produce extensive analyses of the SPES facility in the residual activity viewpoint. 
M. Ferrari et al., The SPES facility as an intense neutron source..., Rad. Applic., 2018, 3, 2, 98-105

\section{CONCLUSION}

Several technological challenges are related to the management of the SPES facility and of its Target Unit as an intense neutron source. The choice of the polymeric components to be used in a neutron radiation environment is of utmost importance for the facility construction and management. Irradiation and testing protocols dedicated to elastomeric O-rings and lubricating greases to be used in SPES have been developed. Commercially available products have been irradiated in a mixed neutron fields and comparatively evaluated. Based on the evolution of standard mechanical parameters as a function of the dose, the most stable products have been selected for SPES application. The experimental and calculation outcomes of a case study dedicated to a selected EPDM-based vacuum O-ring provide useful indications of the usability limits of a SPES gate valve. These results are significant for the SPES construction, providing practical indications for the design of irradiated components.

Monte Carlo simulations of SPES are used to simulate the residual activity of the TIS unit during its life cycle. The outcomes of a multi-step FLUKA simulation allow the temporary storage of the exhausted TIS unit to be designed. Moreover, the residual activity of the entire facility is calculated to plan human maintenance during shutdown periods.

Acknowledgements: The materials science study reported here is supported by the European Spallation Source (ESS) ERIC too.

\section{REFERENCES}

1. G. Prete et al., "The SPES project at the INFNLaboratori nazionali di Legnaro," EPJ Web Conf., vol. 66, 11030, Mar. 2014. DOI: 10.1051/epjconf/20146611030

2. A. Andrighetto et al., "Multifoil UCx target for the SPES project - An update," Eur. Phys. J. A, vol. 30, no. 3, pp. $591-601$, Dec. 2006. DOI: $10.1140 /$ epja/i2006-10144-3

3. A. Monetti et al., "The RIB production target for the SPES project," Eur. Phys. J. A, vol. 51, 128, Oct. 2015. DOI: 10.1140/epja/i2015-15128-6

4. R. O. Bolt, J. G. Carrol, Radiation Effects on Organic Materials, New York (NY), USA: Academic Press, 1963.

5. A. Zenoni et al., "Radiation resistance of elastomeric O-rings in mixed neutron and gamma fields: Testing methodology and experimental results," Rev. Sci. Instrum., vol. 88, no. 11, 113304, Nov. 2017.
DOI: $10.1063 / 1.5011035$

6. A. Andrighetto et al., "SPES: An intense source of Neutron-Rich Radioactive Beams at Legnaro," IOP Conf. Series: J. Phys. Conf. Ser., vol. 966, 012028, 2018.

DOI: $10.1088 / 1742-6596 / 966 / 1 / 012028$

7. M. Ferrari et al., "An Irradiation Campaign of Lubricants at TRIGA Mark II Nuclear Reactor for the European Spallation Source (ESS) and the Selective Production of Exotic Species (SPES) facilities," in Proc. $26^{\text {th }}$ Int. Conf. Nuclear Energy for New Europe (NENE 2017), Bled, Slovenia, 2017, pp. 301.1 - 301.8 .

Retrieved from: https://www.djs.si/proc/nene2017/ht $\mathrm{ml} / \mathrm{pdf} / \mathrm{NENE} 2017$ all.pdf

Retrieved on: Jun. 13, 2018

8. D. Battini et al., "Experimental testing and numerical simulations for life prediction of gate valve O-rings exposed to mixed neutron and gamma fields," unpublished.

9. R. M. Mortier, M. F. Fox, S. T. Orszulik, "Lubricating grease," in Chemistry and Technology of lubricants, 3rd ed., Springer, 2010, ch. 14, pp. $411-432$. DOI: $10.1007 / 978-1-4020-8662-5$

10. MCNPXTM version 2.7.o, Radiation Safety Information Computational Center, Oak Ridge (TN), USA, 2011. Retrieved from: https://rsicc.ornl.gov/; Retrieved on: Jun. 14, 2018

11. M. Ferrari et al., A residual activation study on the SPES Front-End: Dosimetry and Radiation Protection Calculations, SPES-Note-WPBo6 04 0004, Istituto Nazionale di Fisica Nucleare, Laboratori Nazionali di Legnaro, Italy, 2017.

12. A. Ferrari, P. R. Sala, A. Fasso, J. Ranft, FLUKA: A Multi-Particle Transport Code," SLAC-R-773, CERN, Switzerland, 2005.

Retrieved from: https://www.fluka.org/fluka.php?id=s ecured intro;

Retrieved on: Jun. 14, 2018

13. F. X. Gallmeier et al., "The CINDER'9o transmutation code package for use in accelerator applications in combination with MCNPX," in $19^{\text {th }}$ Meeting on Collaboration of Advanced Neutron Sources (ICANS $X I X)$, Grindelwald, Switzerland, 2010.

Retrieved from:

http://www.oecd-nea.org/tools/abstract/detail/CCCo755/;

Retrieved on: Jun. 14, 2018

14. D. Chiesa, "Development and Experimental Validation of a Monte Carlo Simulation Model for the TRIGA Mark II Reactor," Ph.D. dissertation, Università degli Studi di Milano Bicocca, Milan, Italy, 2013.

15. S. Pandini et al., "Effect of combined gamma and neutron irradiation on EPDM and FPM elastomers" in AIP Conference Proceedings, 1981, 020052 (2018) DOI: $10.1063 / 1.5045914$ 